\title{
Comparison of Weight and Body Composition After Gastrectomy Between Elderly and Non-elderly Patients With Gastric Cancer
}

\author{
TORU AOYAMA ${ }^{1,2^{*}}$, YUKIO MAEZAWA $^{1,2^{*}}$, TAKAKI YOSHIKAWA ${ }^{1,2}$, KENKI SEGAMI $^{1,2}$, \\ KAZUKI KANO $^{1,2}$, TSUTOMU HAYASHI ${ }^{1,2}$, TAKANOBU YAMADA ${ }^{1,2}$, MASAKATSU NUMATA ${ }^{2}$, \\ MOTOHICO GODA ${ }^{2}$, HIROSHI TAMAGAWA ${ }^{2}$, TSUTOMU SATO $^{1,2}$, NORIO YUKAWA ${ }^{2}$, \\ YASUSHI RINO ${ }^{2}$, MUNETAKA MASUDA ${ }^{2}$, TAKASHI OGATA ${ }^{1}$, HARUHIKO CHO $^{1}$ and TAKASHI OSHIMA ${ }^{1,2}$ \\ ${ }^{1}$ Department of Gastrointestinal Surgery, Kanagawa Cancer Center, Yokohama, Japan; \\ ${ }^{2}$ Department of Surgery, Yokohama City University, Yokohama, Japan
}

\begin{abstract}
Background: Body weight, especially lean body mass, significantly decreases after gastrectomy for gastric cancer due to surgical invasion, reduced food intake, and reduced mobility, which can reduce the quality of life and induce associated toxicity or reduce compliance with adjuvant chemotherapy. Such risks can be particularly high in elderly patients with gastric cancer. However, whether or not changes in the weight and body composition differ between elderly and non-elderly patients remains unclear. Patients and Methods: This retrospective study examined patients who underwent curative surgery for gastric cancer between May 2010 and February 2017. Body weight and composition were evaluated by a bioelectrical impedance analyzer within 1 week before surgery, at 1 week after surgery, and at 1 and at 3 months after surgery. Patients were classified as elderly ( $\geq 80$ years) or non-elderly ( $<80$ years). Results: Eight-hundred and eighty-eight patients ( 84 elderly and 804 non-elderly) were entered into the present study. Patient background, surgical and clinicopathological factors, and surgical complications did not significantly differ between the two groups. Body weight loss at 1 week, and at 1 and 3 months after surgery,
\end{abstract}

This article is freely accessible online.

*These Authors contributed equally to this study.

Correspondence to: Toru Aoyama, Department of Gastrointestinal Surgery, Kanagawa Cancer Center, 2-3-2 Nakao, Asahi-ku, Yokohama 241-8515, Japan. Tel: +81 455202222, e-mail: t-aoyama@lilac.plala.or.jp and Takaki Yoshikawa, Department of Gastric Surgery, National Cancer Center, 5-1-1 Tsukiji, Chuo-Ku, Tokyo, Japan. E-mail: tayoshik@ncc.go.jp, tel. +81 335422511.

Key Words: Gastric cancer, body weight loss, lean body mass, elderly patients. defined as the decrease from the preoperative value, were $-2.8 \%,-6.5 \%$, and $-9.0 \%$, respectively, in the elderly and $-3.5 \%,-6.0 \%$, and $-8.1 \%$, respectively, in the non-elderly patients $(p=0.111,0.125$, and 0.153 , respectively). The corresponding losses of lean body mass were $-2.6 \%,-6.0 \%$, and $-6.4 \%$, respectively, in the elderly and $-3.5 \%,-4.9 \%$, and $-4.7 \%$, respectively, in the non-elderly patients, with $p$ values of 0.056, 0.036, and 0.029, respectively. Conclusion: Decreases in lean body mass after gastrectomy were greater in elderly than in non-elderly patients. In order to maintain lean body mass among elderly patients, additional care and treatments are needed.

Gastric cancer is the fourth-most common human malignant disease and the second most frequent cause of cancer-related death worldwide (1). Complete resection is essential for the cure of gastric cancer $(2,3)$. Body composition changes are common problems after gastrectomy for gastric cancer and occur during the first 3 months after surgery $(4,5)$. Body composition changes after gastrectomy may occur through various mechanisms, such as a lack of exercise, decrease in food intake, and hypercatabolism associated with inflammatory reactions due to surgical stress. Previous studies have shown that changes in body composition reduced the nutritional status, postoperative quality of life, and compliance with adjuvant chemotherapy, all of which can lead to a poor survival (6-8).

The number of elderly patients has been rapidly increasing worldwide (9). With people of 80 years of age or more representing the fastest growing subset of the population, the management of complex oncological issues promises to become even more challenging. Generally, elderly patients with cancer often have co-morbidities and age-related physiological problems that can lead to greater body composition changes than in non-elderly patients $(10,11)$. 
Furthermore, the rate of muscle protein synthesis is lower in elderly compared to non-elderly adults $(12,13)$. We, therefore, hypothesized that body composition changes after gastrectomy are much more substantial and last much longer in elderly patients than in younger ones, even among patients under similar surgical stress with a similar fast-track program after surgery. If changes in body composition after gastrectomy are indeed much more substantial in elderly patients than in younger ones, additional care or treatments might be needed for elderly patients compared to younger patients.

To test our hypothesis, we performed serial evaluations of the body composition changes after gastrectomy using a bioelectrical impedance analyzer and compared the degree and duration of changes in body composition between elderly and non-elderly patients who underwent curative gastrectomy.

\section{Patients and Methods}

Patients. This was a retrospective cohort study. Patient records were retrieved from a prospectively collected database of Kanagawa Cancer Center from May 2010 to February 2017. The inclusion criteria were as follows: (i) underwent curative gastrectomy with lymph node dissection for gastric cancer as primary treatment, (ii) R0 resection was achieved, (iii) did not experience weight loss over $15 \%$ before surgery, and (iv) a body composition analysis was performed within 1 week before surgery, and at 1 week, 1 month and at 3 months after surgery.

Surgical procedure. All patients underwent distal or total gastrectomy with nodal dissection for gastric cancer. In principle, D1 or a D1+ lymphadenectomy was indicated for cT1N0 tumors, and D2 for $\mathrm{cN}+$ or cT2-T4 tumors regardless of the approach. Spleen-preserving D2 total gastrectomy was permitted in this study.

Perioperative care. Patients received the enhanced recovery after surgery (ERAS) protocol after gastrectomy. The details of this protocol have been reported elsewhere $(14,15)$. In brief, the patients were allowed to eat until midnight on the day before surgery and were required to drink a rehydration solution by 3 hours before surgery. The nasogastric tube was removed immediately after surgery. Oral intake was initiated on postoperative day (POD) 1, beginning with water and an oral nutritional supplement. The patients began to eat solid food on POD 2, starting with rice gruel and soft food on POD 3 and advancing in three steps to regular food intake on POD 7. The patients were discharged when they had achieved adequate pain relief and soft food intake and exhibited normal laboratory data on POD 7. No postoperative medications were used to increase appetite, bowel movement or nutrition supplement for patients in this study. Moreover, in the present study, although all the patients received the same aftercare, such as physical examination and laboratory testing, in the first 3 months after surgery in the Outpatient Clinic, they did not receive oral nutrition supplements to avoid lean mass loss.
Evaluation of operative morbidity and mortality. surgical and nonsurgical complications were assessed prospectively and classified according to the Clavien-Dindo classification (16). Operative mortality was defined as postoperative death from any cause within 30 days after surgery or during the same hospital stay.

Body composition analysis. The segmental body composition was analyzed using a Tanita MC-190EM bioelectrical impedance analyzer (Tanita, Tokyo, Japan), which provides relative information regarding the amount of lean and fat tissue in the trunk area and each limb, as well as the overall body composition and hydration status. The body weight and composition were evaluated by bioelectrical impedance analyzer within 1 week before surgery, and at 1 week, 1 month and 3 months after surgery.

Evaluation, statistical analyses, and ethics. Data values are expressed as the median and range. Statistical analyses were performed using the Chi-square test or the Wilcoxon signed-rank test. A $p$-value of less than 0.05 was considered to indicate statistical significance. The SPSS software package (v12.0 J Win; SPSS, Chicago, IL, USA) was used for all statistical analyses. The R-category and extent of dissection were determined by the Japanese Classification of Gastric Carcinoma, third English edition (17) and the Japanese Gastric Cancer Association guidelines (18). The study was approved by the Institutional Review Board of Kanagawa Cancer Center (2018 Epidemiology-30). The study was conducted in accordance with the Declaration of Helsinki. All participants signed consent forms.

\section{Results}

Background characteristics. Eight hundred and eighty-eight patients were eligible for the present study. The median age was 68 (range=27-90) years. Five-hundred and ninety-five patients were male, and 293 were female. Eight hundred and four patients were classified as non-elderly patients (nonelderly group) and 84 as elderly patients (elderly group). The background characteristics of the patients are summarized in Table I. The American Society of Anesthesiology score was significantly worse and the incidence of the hypertension and chronic obstructive pulmonary disease significantly higher in the elderly group than in the non-elderly group $(p<0.001$, $p=0.001$, and $p=0.001$, respectively). Furthermore, preoperative total body weight and lean body mass was significantly lower in the elderly group $(p=0.003$ and $p<0.001$, respectively). The operative details and pathological findings are shown in Table II. The operative details and pathological findings were similar for the two groups.

Postoperative surgical complications. The details of the postoperative surgical complications are shown in Table III. The overall complication rates were $17.4 \%$ in the non-elderly group and $19.0 \%$ in the elderly group. There were no significant differences between the two groups $(p=0.708)$. Pancreatic fistula and anatomic leakage were the most frequently diagnosed complications in the non-elderly group, followed by 
Table I. Comparison of the patient backgrounds.

\begin{tabular}{|c|c|c|c|c|c|}
\hline & Subgroup & $\begin{array}{l}\text { All cases } \\
\mathrm{N}=888\end{array}$ & $\begin{array}{c}\text { Non-elderly }(<80 \text { years }) \\
\mathrm{N}=804\end{array}$ & $\begin{array}{c}\text { Elderly ( } \geq 80 \text { years) } \\
\qquad \mathrm{N}=84\end{array}$ & $p$-VaIue \\
\hline Age, years & Median (range) & & & & - \\
\hline \multirow[t]{2}{*}{ Gender, n (\%) } & Male & $595(67.0 \%))$ & $540(67.2 \%)$ & $55(65.5 \%)$ & 0.754 \\
\hline & Female & $293(33.0 \%$ & $264(32.8 \%)$ & $29(34.5 \%)$ & \\
\hline \multirow[t]{3}{*}{ ASA-PS, n (\%) } & 1 & $201(22.6 \%)$ & $196(24.4 \%)$ & $5(6.0 \%)$ & $<0.001$ \\
\hline & 2 & $671(75.6 \%)$ & $593(73.8 \%)$ & $78(92.9 \%)$ & \\
\hline & 3 & $16(1.8 \%)$ & $15(1.9 \%)$ & $1(1.1 \%)$ & \\
\hline Total body weight, $\mathrm{kg}$ & Median (range) & $58.5(31.8-102.5)$ & $58.9(31.8-102.5)$ & $56.0(36.1-73.9)$ & 0.003 \\
\hline Lean body mass, $\mathrm{kg}$ & Median (range) & $46.1(27.2-67)$ & $46.9(27.7-67)$ & $42.9(27.2-60)$ & $<0.001$ \\
\hline Body mass index, $\mathrm{kg} / \mathrm{m}^{2}$ & Median (range) & $22.2(13.9-35.6)$ & $22.2(13.9-35.6)$ & $21.8(17.3-28.9)$ & 0.892 \\
\hline \multirow[t]{3}{*}{ Tumor site, n (\%) } & Upper third & $202(22.7 \%)$ & $183(22.8 \%)$ & $19(22.6 \%)$ & 0.003 \\
\hline & Middle third & $429(48.4 \%)$ & $401(49.9 \%)$ & $28(33.4 \%)$ & \\
\hline & Lower third & $257(28.9 \%)$ & $220(27.3 \%)$ & $37(44.0 \%)$ & \\
\hline \multirow[t]{2}{*}{ Clinical T factor, n (\%) } & $\mathrm{T} 1-\mathrm{T} 2$ & $576(64.9 \%)$ & $527(65.5 \%)$ & $49(58.3 \%)$ & 0.188 \\
\hline & $\mathrm{T} 3-\mathrm{T} 4$ & $312(35.1 \%)$ & $277(34.5 \%)$ & $35(41.7 \%)$ & \\
\hline \multirow[t]{2}{*}{ Clinical $\mathrm{N}$ factor, n $(\%)$} & Negative & $704(79.3 \%)$ & $642(79.9 \%)$ & $62(73.8 \%)$ & \\
\hline & Positive & $184(20.7 \%)$ & $162(20.1 \%)$ & $22(26.2 \%)$ & 0.194 \\
\hline \multirow[t]{3}{*}{ Co-morbidity, n (\%) } & Hypertension & $413(46.5 \%)$ & $359(44.7 \%)$ & $54(64.2 \%)$ & 0.001 \\
\hline & Diabetes mellitus & $107(12.0 \%)$ & $93(11.6 \%)$ & $14(16.7 \%)$ & 0.518 \\
\hline & COPD & $176(19.8 \%)$ & $148(18.4 \%)$ & $28(33.3 \%)$ & 0.001 \\
\hline \multirow[t]{2}{*}{ Smoking habit, n (\%) } & Yes & $443(49.9 \%)$ & $404(50.2 \%)$ & $39(46.4 \%)$ & 0.819 \\
\hline & No & $445(50.1 \%)$ & $400(49.8 \%)$ & $45(53.6 \%)$ & \\
\hline
\end{tabular}

ASA-PS: American Society of Anesthesiologists physical status, COPD: chronic obstructive pulmonary disease.

Table II. Surgical and pathological outcomes.

\begin{tabular}{|c|c|c|c|c|c|}
\hline & Subgroup & All cases & $\begin{array}{c}\text { Non-elderly }(<80 \text { years }) \\
\text { N }(\%)\end{array}$ & $\begin{array}{c}\text { Elderly ( } \geq 80 \text { years) } \\
\mathrm{N}(\%)\end{array}$ & $p$-VaIue \\
\hline \multirow[t]{2}{*}{ Lymph node dissection, $\mathrm{n}$} & $\mathrm{D} 1+$ dissection & $540(60.8 \%)$ & $491(61.6 \%)$ & $49(58.3 \%)$ & \multirow[t]{2}{*}{0.468} \\
\hline & D2 dissection & $348(39.2 \%)$ & $313(38.4 \%)$ & $35(41.7 \%)$ & \\
\hline \multirow[t]{3}{*}{ Type of gastrectomy, $n$} & Distal gastrectomy & $558(62.8 \%)$ & $499(62.1 \%)$ & $59(70.2 \%)$ & \multirow[t]{3}{*}{0.348} \\
\hline & Total gastrectomy & $293(33.0 \%)$ & $272(33.8 \%)$ & $21(25 \%)$ & \\
\hline & Other & $37(4.2 \%)$ & $33(4.1 \%)$ & $4(4.8 \%)$ & \\
\hline \multirow[t]{2}{*}{ Type of approach, $n$} & Conventional & $531(59.8 \%)$ & $481(59.8 \%)$ & $50(59.5 \%)$ & \multirow[t]{2}{*}{0.564} \\
\hline & Laparoscopic & $357(40.2 \%)$ & $323(40.2 \%)$ & $34(40.5 \%)$ & \\
\hline Bleeding, $g$ & Median (range) & $130(5-1190)$ & $130(5-1190)$ & $140(5-1050)$ & 0.788 \\
\hline Operative time, $\min$ & Median (range) & $186(81-394)$ & $187(93-394)$ & $173(81-293)$ & 0.652 \\
\hline Harvested lymph nodes, $n$ & Median (range) & $42(9-112)$ & $42(13-110)$ & $40.5(9-112)$ & 0.845 \\
\hline \multirow[t]{4}{*}{ Pathological $\mathrm{T}$ factor, $\mathrm{n}$} & T0-T1 & $519(58.4 \%)$ & $475(59.1 \%)$ & $44(52.4 \%)$ & \multirow[t]{4}{*}{0.104} \\
\hline & $\mathrm{T} 2$ & $109(12.3 \%)$ & $94(11.7 \%)$ & $15(17.8 \%)$ & \\
\hline & $\mathrm{T} 3$ & $111(12.5 \%)$ & $99(12.3 \%)$ & $12(14.3 \%)$ & \\
\hline & $\mathrm{T} 4$ & $149(16.8 \%)$ & $136(16.9 \%)$ & $13(15.5 \%)$ & \\
\hline \multirow[t]{4}{*}{ Pathological $\mathrm{N}$ factor, $\mathrm{n}$} & No & $620(69.8 \%)$ & $568(70.6 \%)$ & $52(61.9 \%)$ & \multirow[t]{4}{*}{0.252} \\
\hline & $\mathrm{N} 1$ & $101(11.4)$ & $94(11.7 \%)$ & $7(8.3 \%)$ & \\
\hline & N2 & $85(9.6 \%)$ & $73(9.1 \%)$ & $12(14.3 \%)$ & \\
\hline & N3 & $82(9.2 \%)$ & $69(8.6 \%)$ & $13(15.5 \%)$ & \\
\hline
\end{tabular}

pneumonia. In contrast, ileus was the most frequently diagnosed complication in the elderly group, followed by pancreatic fistula and pneumonia. Surgical mortality was observed in one patient due to anastomotic leakage and cardiovascular disease in the non-elderly group $(0.1 \%)$ and in two patients due to cardiovascular disease in the elderly group (1.2\%). There were marginally significant differences in surgical mortality between the two groups $(p=0.05)$. 
Table III. Comparison of morbidity and mortality.

\begin{tabular}{|c|c|c|c|c|}
\hline & Grade & Non-elderly (<80 years), N (\%) & Elderly ( $\geq 80$ years), $\mathrm{N}(\%)$ & Total $(\mathrm{N}=888)$ \\
\hline \multirow[t]{4}{*}{ Pancreatic fistula } & 2 & $29(3.6 \%)$ & $5(5.9 \%)$ & $34(3.8 \%)$ \\
\hline & $3 a / 3 b$ & $4(0.5 \%)$ & $0(0 \%)$ & $4(0.4 \%)$ \\
\hline & $4 a / 4 b$ & $1(0.1 \%)$ & $0(0 \%)$ & $1(0.1 \%)$ \\
\hline & 5 & $0(0 \%)$ & $0(0 \%)$ & $0(0 \%)$ \\
\hline \multirow[t]{4}{*}{ Abdominal abscess } & 2 & $6(0.7 \%)$ & $0(0 \%)$ & $6(0.6 \%)$ \\
\hline & $3 a / 3 b$ & $10(1.2 \%)$ & $1(1.2 \%)$ & $11(1.2 \%)$ \\
\hline & $4 a / 4 b$ & $2(0.2 \%)$ & $0(0 \%)$ & $2(0.2 \%)$ \\
\hline & 5 & $0(0 \%)$ & $0(0 \%)$ & $0(0 \%)$ \\
\hline \multirow[t]{4}{*}{ Anastomotic leakage } & 2 & $6(0.7 \%)$ & $0(0 \%)$ & $6(0.6 \%)$ \\
\hline & $3 a / 3 b$ & $21(2.6 \%)$ & $1(1.2 \%)$ & $22(2.4 \%)$ \\
\hline & $4 a / 4 b$ & $4(0.5 \%)$ & $1(1.2 \%)$ & $5(0.5 \%)$ \\
\hline & 5 & $0(0 \%)$ & $0(0 \%)$ & $0(0 \%)$ \\
\hline \multirow[t]{4}{*}{ Anastomotic stenosis } & 2 & $1(0.1 \%)$ & $0(0 \%)$ & $1(0.1 \%)$ \\
\hline & $3 a / 3 b$ & $9(1.1 \%)$ & $1(1.2 \%)$ & $10(1.1 \%)$ \\
\hline & $4 a / 4 b$ & $0(0 \%)$ & $0(0 \%)$ & $0(0 \%)$ \\
\hline & 5 & $0(0 \%)$ & $0(0 \%)$ & $0(0 \%)$ \\
\hline \multirow[t]{4}{*}{ Ileus } & 2 & $5(0.6 \%)$ & $2(2.4 \%)$ & $7(0.7 \%)$ \\
\hline & $3 a / 3 b$ & $9(1.1 \%)$ & $3(3.6 \%)$ & $12(1.3 \%)$ \\
\hline & $4 a / 4 b$ & $0(0 \%)$ & $1(1.2 \%)$ & $1(0.1 \%)$ \\
\hline & 5 & $0(0 \%)$ & $0(0 \%)$ & $0(0 \%)$ \\
\hline \multirow[t]{4}{*}{ Pneumonia } & 2 & $18(2.2 \%)$ & $2(2.4 \%)$ & $20(2.2 \%)$ \\
\hline & $3 a / 3 b$ & $2(0.2 \%)$ & $0(0 \%)$ & $2(0.2 \%)$ \\
\hline & $4 a / 4 b$ & $1(0.1 \%)$ & $0(0 \%)$ & $1(0.1 \%)$ \\
\hline & 5 & $0(0 \%)$ & $0(0 \%)$ & $0(0 \%)$ \\
\hline \multirow[t]{4}{*}{ Postoperative bleeding } & 2 & $4(0.5 \%)$ & $0(0 \%)$ & $4(0.4 \%)$ \\
\hline & $3 a / 3 b$ & $3(0.4 \%)$ & $0(0 \%)$ & $3(0.3 \%)$ \\
\hline & $4 a / 4 b$ & $1(0.1 \%)$ & $0(0 \%)$ & $1(0.1 \%)$ \\
\hline & 5 & $0(0 \%)$ & $0(0 \%)$ & $0(0 \%)$ \\
\hline \multirow[t]{4}{*}{ Wound abscess } & 2 & $4(0.5 \%)$ & $1(1.2 \%)$ & $5(0.5 \%)$ \\
\hline & $3 a / 3 b$ & $0(0 \%)$ & $0(0 \%)$ & $0(0 \%)$ \\
\hline & $4 a / 4 b$ & $0(0 \%)$ & $0(0 \%)$ & $0(0 \%)$ \\
\hline & 5 & $0(0 \%)$ & $0(0 \%)$ & $0(0 \%)$ \\
\hline \multirow{4}{*}{ Pleural effusion } & 2 & $5(0.6 \%)$ & $0(0 \%)$ & $5(0.5 \%)$ \\
\hline & $3 a / 3 b$ & $2(0.2 \%)$ & $1(1.2 \%)$ & $3(0.3 \%)$ \\
\hline & $4 a / 4 b$ & $0(0 \%)$ & $0(0 \%)$ & $0(0 \%)$ \\
\hline & 5 & $0(0 \%)$ & $0(0 \%)$ & $0(0 \%)$ \\
\hline \multirow[t]{4}{*}{ Other } & 2 & $10(1.2 \%)$ & $0(0 \%)$ & $10(1.1 \%)$ \\
\hline & $3 a / 3 b$ & $0(0 \%)$ & $0(0 \%)$ & $0(0 \%)$ \\
\hline & $4 a / 4 b$ & $1(0.1 \%)$ & $0(0 \%)$ & $1(0.1 \%)$ \\
\hline & 5 & $1(0.1 \%)$ & $1(1.2 \%)$ & $2(0.2 \%)$ \\
\hline
\end{tabular}

Body composition. The loss of body weight at 1 week, and 1 and 3 months was $-3.4 \%$ (range $=-14.6 \%$ to $7.2 \%$ ), $-6.1 \%$ (range $=-23.7 \%$ to $5.9 \%$ ), and $-8.3 \%$ (range $=-26.6 \%$ to $7.3 \%$ ) for the whole cohort. Figure 1 shows a comparison of the loss of body weight between the non-elderly and elderly groups at 1 week to 3 months. Marginally significant decreases were observed at 1 and 3 months in the elderly group ( $p=0.125$ and 0.153 , respectively).

In contrast, the loss of lean body mass at 1 week, and 1 and 3 months were $-3.5 \%$ (range $=-27.0 \%$ to $26.1 \%$ ), $-4.8 \%$ (range $=-20.2 \%$ to $17.3 \%$ ), and $-4.9 \%$ (range $=-23.3 \%$ to $24.9 \%$ ), respectively, for the whole cohort. Figure 2 shows a comparison of the loss of lean body mass between the non-elderly and elderly groups at 1 week to 3 months. Loss was significantly greater in the elderly group at 1 and 3 months ( $p=0.036$ and $p=0.029$, respectively). When comparing the loss of fat mass after gastrectomy between the elderly patients and the non-elderly patients, there were no differences between two groups.

\section{Discussion}

The aim of the present study was to compare the degree and duration of body composition changes between elderly and 


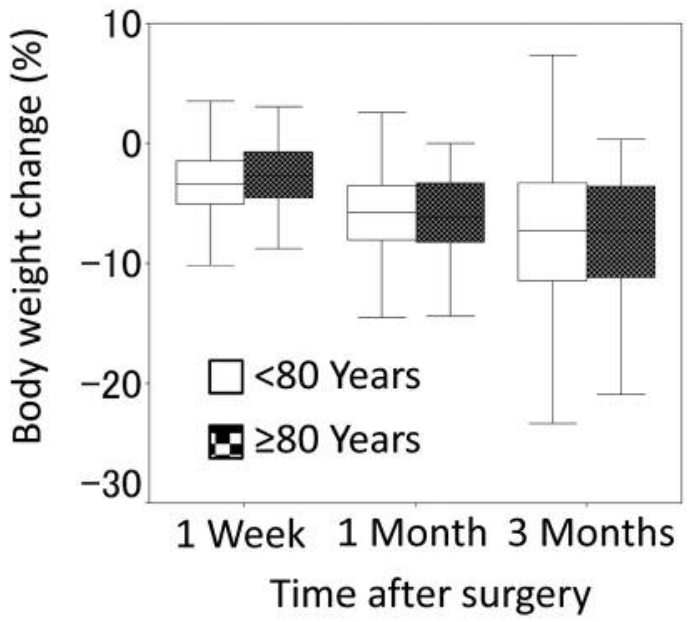

Figure 1. A comparison of the loss of body weight after gastrectomy between elderly and non-elderly patients in this study. Bars: Median, boxes: lower and upper quartiles, lines: minimum and maximum.

non-elderly patients who underwent curative gastrectomy. The major finding was that decreases in lean body mass after gastrectomy were significantly more serious in the elderly patients than in the non-elderly patients at 1 and 3 months after surgery. Our results suggest that additional care or treatment might be needed to maintain lean body mass after gastrectomy in elderly patients.

In the present study, the loss of lean body mass after surgery was significantly greater in elderly patients at 1 and 3 months after surgery. In addition, body weight loss after surgery also tended to be greater in the elderly patients at 1 and 3 months after surgery. Regarding why these results were observed, the first potential explanation is age-related differences in skeletal muscle protein synthesis after surgery (19). Toth et al. examined the effect of age on skeletal muscle protein metabolism by measuring the body composition, muscle function, and muscle protein synthesis in seven young and 15 old volunteers $(72 \pm 1$ years). They found that mixedmuscle and non-myofibrillar protein synthesis tended to be lower in the old volunteers than in the young ones. A similar result had been found in another study (20). Secondly, endocrine reactions to surgical stress have been reported to differ between elderly and non-elderly patients $(21,22)$. When surgical stress occurs, immune cells produce cytokines that act as mediators of both immune and systemic responses to injury. Muscle catabolism is accelerated by the cytokines that are produced during and after surgery, which leads to a decrease in muscle content after surgery. Acute psychological stress activates the endocrine response more profoundly in elderly than in non-elderly patients. In addition, the balance between the catabolic effect of cytokines and the anabolic effect of

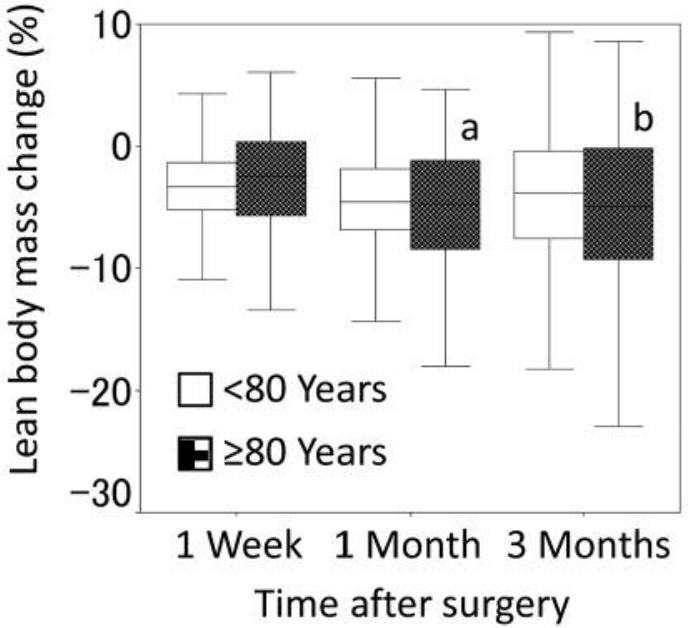

Figure 2. A comparison of the loss of lean body mass after gastrectomy between elderly and non-elderly patients in this study. Significantly different from the non-elderly group at $a_{p}=0.036$, and ${ }^{b} p=0.029$. Bars: Median, boxes: lower and upper quartiles, lines: minimum and maximum.

insulin-like growth factor 1 (IGF1) may play an important role in the loss of lean body mass (21). Aging is also associated with increased cytokine levels/production and reduced circulating IGF1 concentration (22). Thirdly, the elderly patients had already significantly lower preoperative total body weight and lean body mass in the present study. Preoperative loss of total body weight and lean body mass might affect the postoperative finding of greater loss in lean body mass. These factors might affect the differences in the postoperative lean body mass and body weight.

However, previous studies have shown that body weight loss after gastrectomy was approximately $10-20 \%$ of the preoperative body weight, while this value in the present study was $<10 \%$ (5). This discrepancy may be because an ERAS program was used for the perioperative care of gastrectomy patients in this cohort. The ERAS program incorporates a number of factors, including early oral intake and exercise. We standardized the use of the ERAS program in the perioperative care of gastric cancer surgery patients. Our ERAS program includes 13 items. Among them, preoperative counseling, preoperative fasting, and preoperative treatment with carbohydrates prevent nausea and vomiting and stimulate gut motility, while perioperative oral nutrition supported calorie intake. The use of epidural analgesia, early removal of catheters, and mobilization care pathways are useful for ensuring adequate physical activity. Therefore, our ERAS program including the above multiple approaches might be effective for suppressing body weight loss after gastrectomy in both elderly and non-elderly patients. Actually, we previously studied the clinical characteristics, oncological factors, surgical factors, and 
outcomes in patients who underwent elective radical gastrectomy for gastric cancer before and after the introduction of an ERAS protocol. The ratio of the postoperative body weight at 1 week to the preoperative body weight was significantly higher in the ERAS group than in the conventional group (0.95 vs. 0.94, respectively, $p=0.01$ ) (14). If body weight loss during the first month can be suppressed using ERAS care, there is a possibility that subsequent body weight loss might be suppressed.

In order to maintain the lean body mass and body weight loss in elderly patients undergoing curative gastrectomy, optimum care and treatments are needed. Recently, some studies have shown that oral nutritional supplements (ONS) clinically influence the body weight loss in elderly patients. Ohkura et al. evaluated the efficacy of postoperative early intervention with an elemental diet to reduce body weight loss in elderly patients with gastric cancer ( $\geq 80$ years of age). They showed that early intervention with an elemental diet after distal gastrectomy was useful for reducing perioperative weight loss in elderly patients (23). However, they did not evaluate the lean body mass loss. Therefore, the impact of postoperative early intervention with an elemental diet on the lean body mass is unclear.

Hatao et al. investigated the effects of ONS on postoperative body weight loss in patients with gastric cancer who had undergone total gastrectomy or distal gastrectomy (24). In their study, although weight loss in the ONS group after total gastrectomy was significantly less than that in the control group, the weight loss and skeletal muscle mass loss after distal gastrectomy did not significantly differ between the groups. Therefore, whether or not the perioperative use of ONS is actually associated with a reduction in lean body mass loss remains controversial. On the other hand, when we investigated risk factors that affected a lean body mass of 5\% or more after gastrectomy using data from 485 patients, we found that surgical complications with infection or fasting ([odds ratio (OR) 3.576; $p=0.001$ ), total gastrectomy (OR $2.522 ; p=0.0001]$, and male (OR 1.928; $p=0.001$ ) were significant independent risk factors for severe lean body mass loss. We concluded that nutritional intervention or control of surgical invasion should be tested in future clinical trials for gastric cancer patients with these risk factors to maintain lean body mass after gastrectomy. Similar results were observed for body weight loss. These data might help decide what additional care should be taken in the future $(25,26)$.

Several limitations associated with the present study warrant mentioning. Firstly, the present study was a retrospective analysis performed at a single institution. The results need to be confirmed in another cohort or in a prospective multicenter-study. Secondly, segmental body composition was analyzed using a bioelectrical impedance analyzer, which was not able to directly measure muscle mass. The lean body mass is calculated as the sum of the muscle mass and the mass of the visceral organs. However, the mass of the visceral organs would not have been changed by surgery, therefore the major contributor to the change in lean body mass would be a change in the muscle mass. Thirdly, we failed to measure the total calorie intake, physical activity, and serum marker after surgery in the present study. The influence of calorie intake and physical activity on postoperative body composition changes was, therefore, unclear in the present study. Fourthly, the definition of elderly patients is variable. For instance, the United Nations has defined old age as over 60 years of age (27). Conversely, the World Health Organization defined old age as over 65 years of age (28). In this study, we defined elderly patients as those over 80 years of age for the following reasons. The number of elderly patients is rapidly growing worldwide. With people aged 80 and older representing the fastest growing subset of the population, the management of complex surgical issues promises to become even more challenging. However, previous studies and clinical trials excluded patients over the age of 80 years. In addition, generally, elderly patients often have co-morbidities and age-related physiological problems that can lead to avoidance of gastrectomy. Therefore, clinical data including the body composition in elderly patients are scarce. However, the cutoff value may depend on the patient background. In addition, a wide age range (27-80 years) in the 'non-elderly' group would skew and bias the results. Thus, an appropriate cutoff value should be determined in other validation studies in other populations.

\section{Conclusion}

The reduction in lean body mass after gastrectomy was significantly greater in elderly than in non-elderly patients at 1 and 3 months after surgery. Our results suggest that additional care or treatment are needed for elderly patients to maintain lean body mass after gastrectomy.

\section{Availability of Data and Materials}

Data and materials are available to any researcher interested upon reasonable request to the corresponding author.

\section{Conflicts of Interests}

The Authors declare no competing interests in association with the present study

\section{Acknowledgements}

This work was supported, in part, by the non-governmental organization Kanagawa Standard Anti-cancer Therapy Support System. The Authors express their sincere gratitude to Mrs. Natsumi Sato and Mrs. Rika Takahashi for their excellent data management in this study. The present study was presented at the Gastrointestinal Cancers Symposium 2017 (San Francisco, CA, USA). 


\section{References}

1 Torre LA, Bray F, Siegel RL, Ferlay J, Lortet-Tieulent J and Jemal A: Global cancer statistics 2012. CA Cancer J Clin 65: 87108, 2015.

2 Yoshikawa T, Rino Y, Yukawa N, Oshima T, Tsuburaya A and Masuda M: Neoadjuvant chemotherapy for gastric cancer in Japan: a standing position by comparing with adjuvant chemotherapy. Surg Today 44: 11-21, 2014.

3 Aoyama $\mathrm{T}$ and Yoshikawa T: Adjuvant therapy for locally advanced gastric cancer. Surg Today 47(11): 1295-1302, 2017.

4 Adams JF: The clinical and metabolic consequences of total gastrectomy. I: Morbidity, weight, and nutrition. Scand J Gastroenterol 2: 137-149, 1967.

5 Fein M, Fuchs KH, Thalheimer A, Freys SM, Heimbucher J and Thiede A: Long-term benefits of Roux-en-Y pouch reconstruction after total gastrectomy: A randomized trial. Ann Surg 247: 759765,2008

6 Aoyama T, Yoshikawa T, Shirai J, Hayashi T, Yamada T, Tsuchida $\mathrm{K}$, Hasegawa S, Cho H, Yukawa N, Oshima T, Rino Y, Masuda M and Tsuburaya A: Body weight loss after surgery is an independent risk factor for continuation of S-1 adjuvant chemotherapy for gastric cancer. Ann Surg Oncol 20: 2000-2006, 2013.

7 Aoyama T, Sato T, Maezawa Y, Kano K, Hayashi T, Yamada T, Yukawa N, Oshima T, Rino Y, Masuda M, Ogata T, Cho H and Yoshikawa T: Postoperative weight loss leads to poor survival through poor S-1 efficacy in patients with stage II/III gastric cancer. Int J Clin Oncol 22: 476-483, 2017.

8 Aoyama T, Kawabe T, Fujikawa H, Hayashi T, Yamada T, Tsuchida K, Yukawa N, Oshima T, Rino Y, Masuda M, Ogata T, Cho H and Yoshikawa T: Loss of lean body mass as an independent risk factor for continuation of S-1 adjuvant chemotherapy for gastric cancer. Ann Surg Oncol 22: 2560-2566, 2015.

9 The United Nations. Department of Economic and Social Affairs. Population Division. World Population Prospects, the 2015 Revision. Available from: https://esa.un.org/unpd/ wpp/Download/Standard/Population/ (Accessed 17 Mar 2017).

10 Otsuji E, Fujiyama J, Takagi T, Ito T, Kuriu Y, Toma A, Okamoto K, Hagiwara A, Yamagishi H: Results of total gastrectomy with extended lymphadenectomy for gastric cancer in elderly patients. J Surg OncoI 91: 232-236, 2005.

11 Wu CW, Lo SS, Shen KH, Hsieh MC, Lui WY, P'Eng F K: Surgical mortality, survival, and quality of life after resection for gastric cancer in the elderly. W J Surg 24: 465-472, 2000.

12 Lalia AZ, Dasari S, Robinson MM, Abid H, Morse DM, Klaus KA and Lanza IR: Influence of omega-3 fatty acids on skeletal muscle protein metabolism and mitochondrial bioenergetics in older adults. Aging 9: 1096-1129, 2017.

13 Smeuninx B, Mckendry J, Wilson D, Martin U and Breen L: Age-related anabolic resistance of myofibrillar protein synthesis is exacerbated in obese inactive individuals. J Clin Endocrinol Metab 102: 3535-3545, 2017.

14 Yamada T, Hayashi T, Cho H, Yoshikawa T, Taniguchi H, Fukushima $\mathrm{R}$ and Tsuburaya A: Usefulness of enhanced recovery after surgery protocol as compared with conventional perioperative care in gastric surgery. Gastric Cancer 15: 34-41, 2012.

15 Yamada T, Hayashi T, Aoyama T, Shirai J, Fujikawa H, Cho H, Yoshikawa T, Rino Y, Masuda M, Taniguchi H, Fukushima R and Tsuburaya A: Feasibility of enhanced recovery after surgery in gastric surgery: a retrospective study. BMC Surg 14: 41, 2014.
16 Dindo D, Demartines $\mathrm{N}$ and Clavien PA: Classification of surgical complications: a new proposal with evaluation in a cohort of 6336 patients and results of a survey. Ann Surg 240: 205-213, 2004

17 Japanese Gastric Cancer Association: Japanese Classification of Gastric Carcinoma: 3rd English edition. Gastric Cancer 14: 101112,2011

18 Japanese Gastric Cancer Association: Japanese Gastric Cancer Treatment Guidelines 2010 (ver 3). Gastric Cancer 14: 113-123, 2011.

19 Toth MJ, Matthews DE, Tracy RP and Previs MJ: Age-related differences in skeletal muscle protein synthesis: relation to markers of immune activation. Am J Physiol Endocrinol Metab 288: E883-891, 2005.

20 Tanner RE, Brunker LB, Agergaard J, Barrows KM, Briggs RA, Kwon OS, Young LM, Hopkins PN, Volpi E, Marcus RL, LaStayo PC and Drummond MJ: Age-related differences in lean mass, protein synthesis and skeletal muscle markers of proteolysis after bed rest and exercise rehabilitation. J Physiol 593: 4259-4273, 2015.

21 Copeland KC, Colletti RB, Devlin JT, and McAuliffe TL: The relationship between insulin-like growth factor-1 and aging. Metabolism 39: 584-587, 1990.

22 Roubenoff R, Harris TB, Abad LW, Wilson PWF, Dallal GE, and Dinarello CA: Moncyte cytokine production in an elderly population: effect of age and inflammation. J Gerontol 53A: M20-M26 1998.

23 Ohkura Y, Haruta S, Tanaka T, Ueno $\mathrm{M}$ and Udagawa $\mathrm{H}$ : Effectiveness of postoperative elemental diet (Elental ${ }^{\circledR}$ ) in elderly patients after gastrectomy. World J Surg Oncol 14: 268, 2016.

24 Hatao F, Chen KY, Wu JM, Wang MY, Aikou S, Onoyama H, Shimizu N, Fukatsu K, Seto Y and Lin MT: Randomized controlled clinical trial assessing the effects of oral nutritional supplements in postoperative gastric cancer patients. Langenbecks Arch Surg 402: 203-211, 2017.

25 Aoyama T, Sato T, Segami K, Maezawa Y, Kano K, Kawabe T, Fujikawa H, Hayashi T, Yamada T, Tsuchida K, Yukawa N, Oshima T, Rino Y, Masuda M, Ogata T, Cho H and Yoshikawa $\mathrm{T}$ : Risk factors for the loss of lean body mass after gastrectomy for gastric cancer. Ann Surg Oncol 23: 1963-1970, 2016.

26 Segami K, Aoyama T, Kano K, Maezawa Y, Nakajima T, Ikeda K, Sato T, Fujikawa H, Hayashi T, Yamada T, Oshima T, Yukawa N, Rino Y, Masuda M, Ogata T, Cho H and Yoshikawa T: Risk factors for severe weight loss at 1 month after gastrectomy for gastric cancer. Asian J Surg 41: 349-355, 2018.

27 UN (United Nations Population Division). Personal correspondence with Marybeth Weinberger, Chief, Population and Development Section, January 2001.

28 Kowal P and Dowd JE: Definition of an older person. Proposed working definition of an older person in Africa for the MDS Project 2001. Geneva: World Health Organization, 2001. Doi: 10.13140/2.1.5188.9286.
Received October 23, 2018

Revised November 4, 2018

Accepted November 7, 2018 